Voix et Images

volxetimages

\title{
Alain Grandbois ou le procès métaphorique
}

\section{Noël Audet}

Volume 2, numéro 1, septembre 1976

Fernand Leduc

URI : https://id.erudit.org/iderudit/200021ar

DOI : https://doi.org/10.7202/200021ar

Aller au sommaire du numéro

\section{Éditeur(s)}

Les Presses de l'Université du Québec

\section{ISSN}

0318-9201 (imprimé)

1705-933X (numérique)

Découvrir la revue

\section{Citer cet article}

Audet, N. (1976). Alain Grandbois ou le procès métaphorique. Voix et Images, 2(1), 60-70. https://doi.org/10.7202/200021ar d'utilisation que vous pouvez consulter en ligne.

https://apropos.erudit.org/fr/usagers/politique-dutilisation/ 


\section{Alain Grandbois ou le procès métaphorique}

Dans un article précédent ${ }^{1}$, on aura vu comment, chez Saint-Denys Garneau, la signification se construisait en glissant de syntagme en syntagme, en se déplaçant le long du canal étroit de la contiguïté, comme l'eau envahirait un tissu (textuel) selon les lois de la capillarité, sans rupture. La signification s'y trouve littéralement filée, déplacée de proche en proche, à la dérive sur les syntagmes. Les premiers vers du poème «Poids et mesures ${ }^{2}$, ont l'intérêt d'illustrer ceci sur le plan thématique et sur le plan de l'écriture (procès du sens).

II ne s'agit pas de tirer les choses par les cheveux

D'attacher par les cheveux une femme

D'empiler des morts à la queue-leu-leu

à la queue d'un cheval

Au fil de l'épée, au fil du temps.

Dès le premier vers, l'idiotisme "tirer par les cheveux", qui signifie au sens figuré: exagérer, forcer les conclusions, est pris au sens littéral, c'est-à-dire que la fonction métaphorique en est évacuée au profit du procès métonymique, le poète ne retenant que l'idée de tirer et celle de cheveux (fil). Cette prémisse ne pouvait qu'induire: tirer une femme par les cheveux; de là on glisse à la cause, à ce qui tire (le cheval), sans doute suite à une identification entre cheveux et queue de cheval (sorte de coiffure), ou à un glissement phonologique (cheveux - chevaux). Voilà le second vers construit entièrement par ces glissements. Du deuxième au troisièm ? vers, il me paraît y avoir deux sortes d'enchaînement/déplacement: l'un serait l'effet logique du vers précédent: la mort; l'autre, une transformation par contamination phonétique: queue/queue-leu-leu, où les sèmes d'itérativité et de durativité sont introduits, en plus de l'idée de ligne ou de fil présente depuis le début. Enfin le dernier vers de la strophe est en rapport métonymique avec "morts" du vers précédent (relation de l'effet à la cause) et réduit à son tour les aspects métaphoriques de deux autres idiotismes: fil (tranchant) de l'épée et fil (succession continue) du temps. Dans le contexte, ces expressions reprennent davantage l'idée de ligne (queue-leu-leu) et sont surdéterminées par un rapport de conti- 
guïté: les morts sont empilés le long de... le long d'un fil. Comme la signification.

Avec Alain Grandbois, on observera un processus diamétralement opposé, qui nous permettra de réfléchir sur le fonctionnement métaphorique. Le poème de Grandbois se construit en effet sur une série d'équivalences paradigmatiques, et les rapports métonymiques, s'ils sont présents, ne servent qu'à structurer le champ référentiel et à faire ainsi avancer la signification malgré la pure redondance métaphorique. Notons d'autre part que, contrairement à Saint-Denys Garneau que le procès métonymique forçait à narrativer son discours, chez Alain Grandbois il y aura une nette prédominance du plan descriptif.

\section{UN MODĖLE PARADIGMATIQUE}

Selon Roman Jakobson, l'écriture qui privilégie la similarité (le paradigmatique) marquera moins fortement les relations syntaxiques et l'on observera par conséquent une utilisation moindre des mots à "fonctions purement grammaticales, tels que les conjonctions, prépositions, pronoms et articles ${ }^{3}$,... Pour les mêmes raisons, le texte poétique de ce type recourra aux appositions nombreuses, aux synonymes, aux antonymes et à toute forme de parallélismes phonétiques, syntaxiques et sémantiques.

Le premier poème des îles de la nuit ${ }^{4}$, choisi parce qu'il est le premier, nous permettra de lire l'ensemble du modèle poétique de Grandbois ou l'écriture du paradigme.

\section{Ô TOURMENTS...}

1 Ô tourments plus forts de n'être qu'une seule apparence

Angoisse des fuyantes créations

Prière du désert humilié

Les tempêtes battent en vain vos nuques bleues

Vous possédez l'éternelle dureté des rocs

Et les adorables épées du silence ont en vain défié vos feux noirs

II Tourments sourdes sentinelles

$O$ vous soutes gorgées de désirs d'étoiles

Vos bras d'hier pleins des bras d'aujourd'hui

Ont fait en vain les gestes nécessaires

Vos bras parmi ces éventails de cristal

Vos yeux couchés sur la terre

Et vos doigts tièdes sur nos poitrines aveugles

N'ont créé pour notre solitude qu'une solitude d'acier

III Je sais je sais ne le répétez pas

Vous avez perdu ce dur front de clarté

Vous avez oublié ces frais cheveux du matin

Et parce que chaque jour ne chante plus son passage 
Vous avez cru l'heure immobile et la détresse éteinte

Vous avez pensé qu'une route neuve vous attendait

IV Ó vous pourquoi creuser cette fosse mortelle

Pourquoi pleurer sous les épaules des astres

Pourquoi crier votre nuit déchaînée

Pourquoi vos mains de faible assassin

Bientôt l'ombre nous rejoindra sous ses paupières faciles

Et nous serons comme des tombes sous la grâce des jardins

$v$ Non non je sais votre aventure

Je sais cet élan retrouvant le ciel du mât

Je sais ce corps dépouillé et ces larmes de songe

Je sais l'argile du marbre et la poussière du bronze

Je sais vos sourires de miroirs

Ces genoux usés que ronge la ténèbre

Et ce frisson des reins inaccessible

VI Pourquoi le mur de pierre dites-moi

Pourquoi ce bloc scellé d'amitié

Pourquoi ce baiser de lèvres rouges

Pourquoi ce fiel et ce poison

Les minutes du temps me marquent plus que vos trahisons

VII O navires de hauts-bords avec ce sillage de craie

Vos voiles déployées votre haine se gonfle

Pourquoi creuser ces houles comme une tranchée de sang

Pourquoi ces hommes penchés sur la mer comme aux fontaines de soif

Si les morts de la veille refusent de ressusciter

Le premier vers de la strophe I trace déjà le modèle de la strophe, comme toute la strophe expose le programme du poème en entier. Le premier vers se constitue en effet de deux termes appartenant à deux champs distincts et la strophe se développe en articulant ces deux champs, vers après vers:

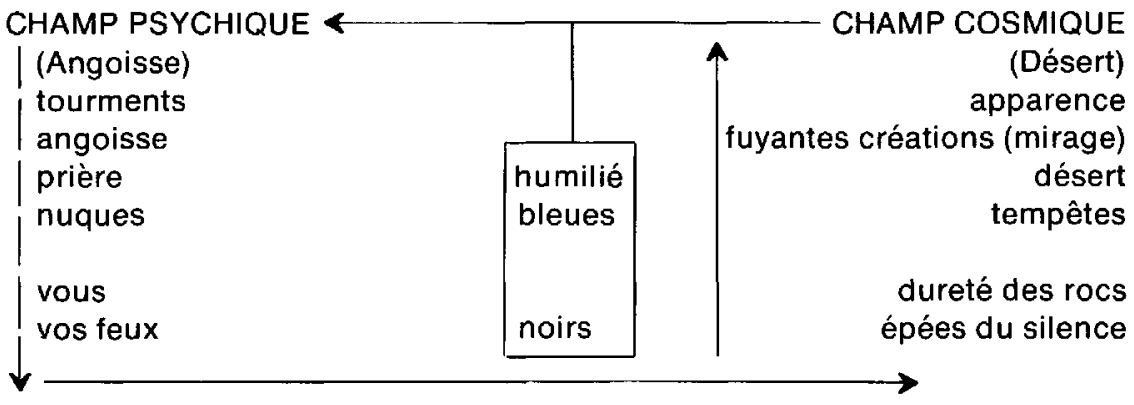


Ce tableau permet de visualiser le parfait équilibre entre les deux séries, dont l'une, celle du champ psychique, illustre parfaitement le principe des équivalences dont parle Jakobson dans ses Essais. La série du champ cosmique offre pour sa part une première difficulté dans le cadre de mon hypothèse. Pour peu que l'on prenne le sémème “désert" comme terme central, on remarque aussitôt que tout ce champ se constitue de métonymies ou plus justement de synecdoques du désert, soit tous les éléments qui lui sont liés par contiguïté spatiale: les mirages (apparence, fuyantes créations), les tempêtes, les rocs, le silence.

Mais revenons au paradigme de l'angoisse afin de mieux définir la nature et la fonction de ces rapports de contiguïté qui semblent contribuer aussi fortement que les rapports de similarité au procès de la signification. Le dictionnaire Robert définit l'angoisse comme «malaise psychique et physique, né du sentiment de l'imminence d'un danger, caractérisé par une crainte diffuse..." En d'autres termes la cause de l'angoisse n'est jamais identifiée comme telle, sinon l'angoisse ferait place à la peur devant un danger réel ou à la sérénité en l'absence d'un tel danger objectif. Or il m'apparaît qu'Alain Grandbois ne fait que définir poétiquement l'angoisse en dépliant le paradigme qui comporte deux attributs principaux: soit la peur, active, murmurante (prière) - devant l'insaisissable, l'innommé, le vide (désert); l'angoisse ne devant cesser que si le vide se connaît comme tel, d'où le sème positif attaché à "silence ": faire taire l'angoisse.

On voit comment le champ cosmique assume l'aspect physique de la définition et permet le procès métaphorique tout en assurant une progression de la description: on était parti d'un terme général (apparence) pour arriver à une métaphore connotant le désespoir («feux noirs» des tourments, ou brûlure sans même la lumière, c'est-à-dire sans la connaissance), en passant par le désert «humilié", soit écrasé physiquement et moralement, et les «tempêtes" des tourments ou possiblement la palpitation du sang pressé à la nuque bleue - blues.

On a donc dans cette première strophe six vers et six définitions redondantes de l'angoisse. Une échelle d'équivalences paradigmatiques à six paliers.

Et les synecdoques du champ cosmique (qui ne se réalisent d'ailleurs pas en figures rhétoriques), est-ce arbitrairement qu'on leur assigne moins d'importance qu'à celles qui structurent les poèmes de SaintDenys Garneau? Quelques précisions s'imposent. En premier lieu, le champ cosmique est ici subordonné au champ psychique; ce dernier appartient à la dénomination, au défini, c'est-à-dire que le sémème «angoisse» est posé, dans sa redondance sémantique même, pour être défini dans/ par le champ cosmique qui appartient donc au définisseur et sert structurellement à une équivalence sémantique. L'intersection des deux champs produit les métaphores horizontales (humilié, bleues, noirs). Les rapports de contiguïté ne président donc pas au progrès de la signifiance (moyen principal de la production du sens) comme chez Saint-Denys Garneau; ils sont associés secondairement à ce procès. Comme on l'a 
vu, c'est en effet le paradigme qui structure la strophe, les six vers se présentant comme six définitions équivalentes.

Il me semble découvrir en outre la fonction des rapports synecdochiques dans les poèmes de Grandbois, rapports beaucoup plus nombreux qu'une première étude ne nous le laisserait penser. A mon avis, ces rapports de contiguïté ont pour fonction de permettre l'avancée du sens puisque l'équivalence, par définition, ne saurait le faire. Le poète n'a plus que l'intensité descriptive pour marquer l'avancée du sens (sorte de graduation qualitative inscrite dans le mouvement du texte). Par exemple, il n'y a pas progrès du sens dans les synonymes tourments, angoisse, prière; mais il y a bien une avancée sémantique de l'autre côté, entre: apparence - fuyantes créations (qui ajoute le sème de mobilité) - désert humilié (qui ajoute les sèmes d'espace indéfini, de lisse et d'abaissement) - tempêtes (dramatisation) — dureté des rocs (sème d'immuabilité) qui induira le désespoir contenu dans - en vain - et - vos feux noirs.

C'est que le découpage synecdochique permet d'abord de séparer le champ en éléments divers et donc d'introduire entre eux, par la suite, un classement des rapports variables, ce que n'autorise pas le saut d'une équivalence globale à une autre.

Enfin pour en terminer avec ce que je qualifierai de fausses synecdoques, examinons l'environnement syntagmatique de chacun de ces éléments du désert. On remarquera vite que la similarité fonctionne non seulement verticalement comme je l'ai montré, mais qu'elle s'étend également sur le plan horizontal et vient écraser le procès métonymique naissant. Soit: seule apparence/fuyantes créations/désert humilié/tempêtes.. (nuques) bleues/dureté des rocs/feux noirs/épées du silence. À partir de ces adjectifs et déterminants, on constate que ce sont là les prédicats de l'angoisse elle-même qui viennent contaminer les déterminants du champ cosmique au lieu de le qualifier comme tel. S'offre donc une nouvelle série paradigmatique en équivalence à l'angoisse. (ANGOISSE = SEULE, solitude; FUYANTES, impossibilité de saisir; HUMILIÉ, domination de l'Autre, négation du moi; BLEUES, en contexte indiquerait la répétition de coup, la fatigue, la tension artérielle, veines bleues; DURETÉ, durativité, permanence du phénomène, sans espoir; NOIRS, absence, mort, deuil, désespoir; ÉPÉES, joue un rôle particulier, sème positif: combat, sèmes négatifs: épée de Damoclès, angoisse.

C'est dire la place minime qu'occupe la procédure métonymique dans cet univers où dominent sans équivoque les rapports de similarité.

A ce stade de l'analyse, on peut faire l'hypothèse, en quelques mots ou images, que le discours poétique d'Alain Grandbois utilise la contiguïté pour l'avancée du sens comme une navette mobile parmi la trame parallèle du tissu textuel. Aucune poésie serait-elle possible sans cette interaction des deux axes: la signification se pose et s'ouvre grâce à la similarité et elle s'avance, se nuance, dans le discours par et à travers les relations de contiguiité. 


\section{LE MODÈLE ÉTENDU AU POÈME}

La seconde strophe recourt au même modèle de développement, à peu de choses près. Toutefois, si le champ psychique demeure le même, il sera posé en équivalence au champ corporel et non plus cosmique. Les rapports synecdochiques seront de nouveau présents avec la même. fonction dans l'économie textuelle.

La strophe débute sous le même signe (tourments) suivi d'équivalences métaphoriques (sourdes sentinelles, soutes) et de la même personnification qui avait été annoncée dans la première strophe par "vos nuques". C'est donc autour du corps cette fois que les rapports métonymiques structureront l'avancée du sens: "Vos bras d'hier pleins des bras d'aujourd'hui/... en vain les gestes... Nos bras parmi.../Vos yeux couchés... /Et vos doigts tièdes..." sans compter le "sourdes sentinelles" (ouïe) du premier vers et le «soutes gorgées..." du second posant le tourment comme privé de l'ouïe dans un cas et de la vue dans l'autre, soit comme pure force intérieure, instinctuelle. C'est pourquoi le silence qu'il n'entend pas ne saurait le faire taire ni les gestes mécaniques l'apaiser.

On a donc le même modèle métaphorique, utilisant des rapports métonymiques pour saturer l'espace de la signification et par le fait même lui faire parcourir une avancée. Dans cette seconde strophe, de «vos bras parmi ces éventails de cristal» à «vos yeux couchés sur la terre" à "vos doigts tieddes sur nos poitrines aveugles", il y a chute parallèle à celle de la première strophe, soit passage du désir démiurgique à la matérialité de l'angoisse (tempêtes/terre) au sentiment d'horreur (feux noirs/doigts tièdes...); encore une fois ce progrès du sens est davantage assuré par les déterminants que par les termes désignant les parties du corps.

Poursuivons le parallèle entre les deux premières strophes. On constate qu'il y a redondance sémantique ou reprise presque terme à terme, la seule difficulté venant du fait qu'un même syntagme dans l'une peut en recouper plusieurs dans l'autre à cause de la pluralité des sens métaphoriques et tellement l'unité paradigmatique est grande.

Ô tourments

STROPHE I

seule apparence

(non vu)

fuyantes créations

prière du désert humilié

tempêtes... nuques bleues

éternelle dureté des rocs

silence

vos feux noirs
Tourments

STROPHE II

sourdes sentinelles

(n'entendent pas)

soutes

(aveugles)

solitude

gorgées de désirs d'étoiles

éventails de cristal

en vain les gestes nécessaires

yeux couchés sur la terre

(chiasme)

solitude d'acier

solitude

doigts tièdes/poitrines aveugles 
Les parallélismes sémantiques ${ }^{5}$ sont suffisamment nombreux pour nous permettre de conclure que la strophe II se pose en équivalence à la première, en d'autres termes comme une région voisine du même paradigme. Dans cette perspective, le poème en entier ne serait que le dépliage et la projection d'un même «domaine" paradigmatique dans le discours. Un repérage sommaire dans les autres strophes nous l'indiquerait déjà de façon convaincante.

Ainsi, en partant de la dernière strophe, « $O$ navires de hauts-bords" (VII) répondrait à «bloc scellé d'amitié» (VI), à «cet élan retrouvant le ciel du mât"(V), à «les épaules des astres" (IV), à «ce dur front de clarté " (III), à «gorgées de désirs d'étoiles» (II, à «fuyantes créations" (I).

De même "fuyantes créations" trouve toute une série de synonymes autres, si on lui donne un autre sens, soit la fragilité de l'art: "sillage de craie" (VII), "Je sais l'argile du marbre et la poussière du bronze" $(V)$, etc. Mais cette analyse complète n'est possible que dans le cadre d'une recherche sur les isotopies ${ }^{6}$ du poème, ce qui échappe à notre présent objet. Disons tout de même que, grâce au fonctionnement métaphorique, ce poème contient trois isotopies ou strates de significations fortement structurées: isotopie du dramé psychique/métaphysique, celle de l'écriture et celle de l'amour. Après une telle étude, le poème apparaîtrait comme archi-paradigme de ces trois domaines de signification.

Pour terminer l'examen de ce poème, je ferai remarquer un dernier aspect. Si dans la strophe I le champ cosmique était dominant, alors que dans la deuxième c'était surtout le champ corporel, les autres strophes vont continuellement mêler les deux champs pour introduire, en dernière strophe, une série de termes marins en rapports de contiguïté avec la mer (navires, sillage, voiles, houles) qui opèrent de la même façon que les synecdoques du désert en première strophe et bouclent parfaitement le poème, tout entier ramassé entre aLes tempêtes battent en vain vos nuques bleues" et "ces houles comme une tranchée de sang". La définition de l'angoisse, en termes de formules équivalentes, n'aura pas progressé entre ces deux points extrêmes, mais grâce aux rapports de contiguïté, elle aura cheminé (syntagmatiquement) du désert, à travers le corps, jusqu'à la mer, en marquant fortement sa redondance métaphorique et en accroissant son intensité dramatique.

\section{LE MOdELLE ET L'OEUVRE}

Ce modèle de fonctionnement, ou ce procès du sens, que je viens de repérer dans le poème «Ô tourments... " de Grandbois est-il généralisable à l'ensemble de l'œuvre? Est-il plus ou moins opérant dans tout texte poétique?

Je ne répondrai ici qu'à la première question. Bien sûr, on trouvera sans doute chez Grandbois des poèmes d'une autre venue, où la signification procèderait davantage de relations métonymiques, où le récit 
s'organiserait au détriment (?) de la métaphore. Dans les îles de la nuit, je n'en vois qu'un plus fortement narrativé et l'exception, si c'en est une, s'expliquerait aisément. II s'agit en effet du dernier poème du recueil “Fermons l'armoire... ", véritable périple intérieur où la dominance complète du métaphorique n'aurait pas su rendre compte du sujet de ce poème terminal. La structure de ce poème procède donc d'un découpage spatiotemporel et le sens semble s'organiser à partir de rapports de contiguïté: "Fermons l'armoire aux sortilèges/ll est trop tard pour tous les jeux/Je sais.../Je vois.../J'ai trop aimé.../J'ai vu.../Rien ne remuera plus dans l'ombre". C'est donc un balayage du passé au futur qui passe en revue tous les lieux thématiques du poète.

Toutefois, à l'intérieur des strophes, quand l'aspect thématique (poésie, amour, mort) est bien amorcé, la métaphore et les échelles paradigmatiques reprennent leur droit. Ainsi, par exemple, dans la première strophe:

Mes yeux sont aveuglés

Mon univers sera englouti avec moi

Je m'enfoncerai dans les cavernes profondes

La nuit m'habitera et ses pièges tragiques

Les voix d'à côté ne me parviendront plus

Je posséderai la surdité du minéral

Tout sera glacé

Et même mon doute?

Sans grand besoin de démonstration, on concevra facilement que ces vers déplient la même région paradigmatique en autant d'équivalences de la mort perçue comme privation des sens: aveuglés / englouti / m'enfoncerai / cavernes / nuit / piège / voix ne me parviendront plus / surdité du minéral / glacé / même mon doute.

Mais revenons à quelques autres poèmes afin de montrer la pertinence du modèle proposé et introduire quelques aspects négligés en cours de route.

Le paradigmatique ne contient pas que des synonymes: il organise également les antonymes. Or une des caractéristiques du style de Grandbois, c'est bien le contraste et le chiasme, soit différentes formes de l'inversion des contenus.

Le poème 2 des illes de la nuit, en plus de reprendre le cheminement dégagé dans le premier poème (équivalences paradigmatiques relayées par des rapports métonymiques), illustre bien cet aspect.

Les tunnels planétaires jouaient le jeu quotidien

Le sang se mêlait à des couleurs jamais perçues

Une fois seulement ce grondement extraordinaire prenait des intimités de seuil de maison

Et c'était le son d'un amour provincial sur les plages de l'aube ${ }^{8}$. 
Nous remarquons que la strophe comporte deux champs, le cosmique et le quotidien, et les pose en contrastes croisés, c'est-à-dire en chiasmes:

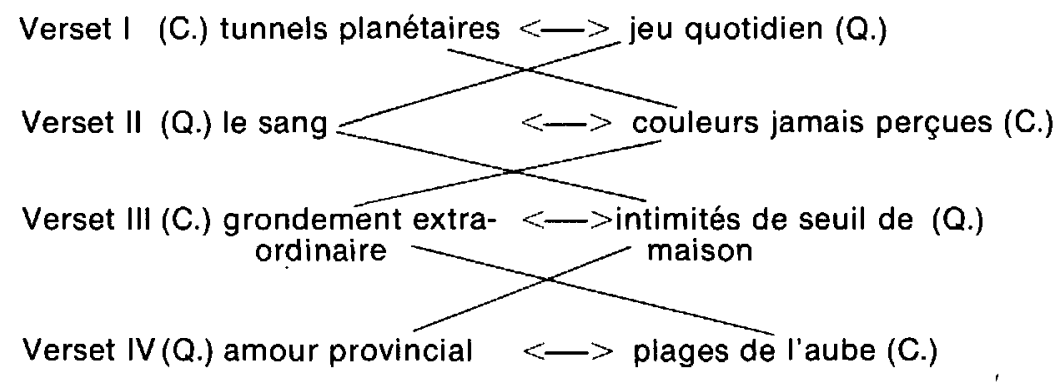

Et le dernier verset: «Tes paumes ouvertes recréaient les destins abolis» scelle l'unité du système.

Le poème 8 , le plus court du recueil, met en scène des sentiments contraires mais dont le désir/rejet est indécidable, c'est-à-dire que tout se passe comme si le poète mêlait les sèmes négatifs et positifs des uns et des autres dans un écheveau habile et inextricable:

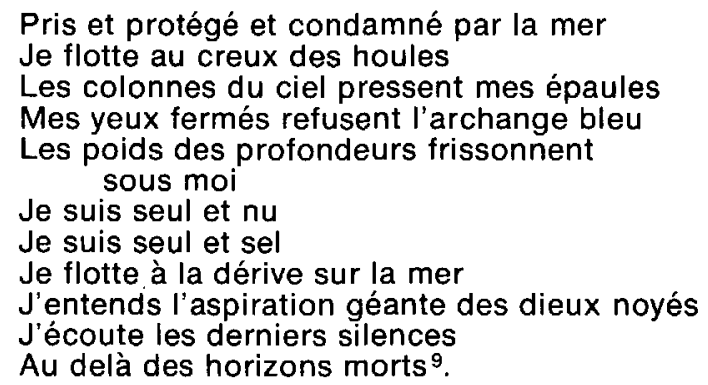

Le programme de tout le poème est tracé dans le premier vers. Le reste du poème ne fait que déployer ce paradigme de l'abandon/défaite (condamnation). L'abandon semble provenir de l'acceptation de la condamnation - protégé parce que condamné par la mer. Le vers 2 , ambigu, contient les deux aspects extrêmes du paradigme: abandon/défaite, désir. Du vers 3 au vers 6 , c'est le rejet du naufrage involontaire. Du vers 6 à la fin, c'est l'abandon ou la fin de la résistance et l'investissement positif de la dérive, ce qui reprojette sur tout le poème une ambiguïté du désir/ rejet. Ici les contraires ne sont plus seulement posés mais confondus, c'est-à-dire rendus au système général des équivalences.

On pourrait multiplier les exemples prouvant la prépondérance du procès métaphorique malgré la fonction de relais des rapports métonymiques (ces derniers fonctionnent comme la coordination et/ou). Le poème 15 «Avec ta robe...", comme d'autres, laisserait croire à un tableau construit sur les rapports de contiguïté. En effet le poème comporte six strophes dont les trois premières décrivent la jeune fille enfant: 
Avec ta robe sur le rocher comme une aile blanche

Des gouttes au creux de ta main comme une blessure fraî́che

Et toi riant la tête renversée comme un enfant seul

Avec tes pieds faibles et nus sur la dure force du rocher

Et tes bras qui t'entourent d'éclairs nonchalants

Et ton genou rond comme l'île de mon enfance

Avec tes jeunes seins qu'un chant muet soulève pour une vaine allégresse

Et les courbes de ton corps plongeant toutes vers ton frêle secret

Et ce pur mystère que ton sang guette pour des nuits futures ${ }^{10}$.

On lit du vers 1 au vers $9^{11}$ la désignation d'un vêtement et de huit parties du corps: ta robe / ta main / ta tête / tes pieds / tes bras / ton genou / tes jeunes seins / les courbes de ton corps / ton frêle secret... Nous sommes donc bel et bien en plein rapport métonymique. Or on ne peut s'en tenir à cette lecture qui n'est que la lecture du champ référentiel. Dans le texte, il ne s'agit pas de neuf métonymies mais bien de neuf métaphores (ou équivalences paradigmatiques) de la candeur, comme l'indique le travail de la syntaxe. Le syntagme, au lieu de construire la métonymie, la détruit en effet et produit la métaphore. "Avec ta robe sur le rocher comme une aile blanche", où aile blanche est en relation métaphorique avec robe (blanche) qui, à son tour, devient (deviennent) métaphore de la candeur; comme "gouttes au creux de la main " et «blessure fraîche», comme "riant la tête renversée", etc. Encore une fois, les neuf vers déplient en autant d'échelons le même paradigme, celui de la candeur que guette l'amour.

Or si les rapports métonymiques (parties du corps) sont si présents dans la première partie de ce poème, c'est justement parce qu'ils agissent également comme complément d'objet direct de la seconde partie, c'està-dire qu'ils se chargent aussi de ce sens logique; "Laisse-moi ne plus te voir " ou voir cela: ta robe, ta main... parce que, le vers final le proclame, vont se refermer sur cela, ta main, ta tête, etc., "Les lourdes portes de l'oubli». Donc c'est un refus de voir, de procéder au découpage métonymique, à la mise en évidence de ces gros plans par peur d'oublier, problème que la métaphore contourne en ouvrant le sens, en lui conférant une qualité abstraite (identité de sèmes) qui le fait échapper à la linéarité du discours aussi bien qu'au temps. La métaphore à ce titre ne serait pas censure mais une sorte de fuite en avant...

On retrouve donc toujours la même procédure à l'œuvre: si les rapports de contiguïté organisent l'exposition du champ et servent de relais 
pour l'avancée du sens, le procès d'écriture lui-même est régi par les rapports de similarité. On a vu que chez Saint-Denys Garneau, au contraire, les rapports de contiguïté régissaient et le développement du thème et le procès, pour finalement produire comme effet de lecture, une série d'équivalences également.

Bien que d'une écriture plus cassée, une brève analyse de Rivages de l'homme et de l'Étoile pourpre semble indiquer la présence du même modèle.

\section{C'était l'ombre aux pas de velours \\ Les étoiles sous le soleil mort \\ Les hommes et les femmes nus \\ La faute n'existait plus ${ }^{12}$.}

Cette première strophe de «l'Étoile pourpre» fonctionne comme le paradigme à quatre termes de l'unité des contrastes (ou réduction des contradictions), sorte d'état de grâce paradisiaque. Chaleur douce, sans violence, lumière tamisée des étoiles (remplaçant le soleil mort), hommes et femmes nus, c'est-à-dire sans contrainte morale, sociale, ni biologique, la Faute (la contradiction avec soi-même) n'existait plus...

N'est-ce pas d'ailleurs une des fonctions mythiques de la poésie que de faire coïncider les opposés dans un même paradigme? Les symbolistes aussi l'ont cru.

(Note: Il serait intéressant de mettre en rapport l'abondance des phrases nominales avec l'écriture métaphorique. L'équivalence d'une phrase à l'autre devient plus apparente du fait que les phrases fonctionnent comme une série de substantifs en apposition.)

Noël Audet

\footnotetext{
1. Voix et images, vol. I, $n^{\circ} 3$, p. 432-441.

2. Saint-Denys Garneau, Poésies complètes, Montréal, Fides, 1949, p. 194.

3. Essais de linguistique générale, Paris, Minuit, 1970, p. 57.

4. Poèmes, Montréal, L'Hexagone, 1973, p. 11-14.

5. Note: II n'y a pas place, dans le cadre de cette publication, pour l'étude des parallélismes syntaxiques et phonémiques qui serait tout aussi pertinente.

6. “On appelle isotopie toute itération d'une unité linguistique." F. Rastier, dans A.J. Greimas et collaborateurs, Essais de sémiotique poétique, Paris, Larousse, 1972, p. 82.

7. Op. cit., p. $93-94$.

8. Ibid., p. 15.

9. Ibid., p. 35.

10. Ibid., p. 48-49.

11. Note: je conçois comme un seul vers l'unité qui commence par une majuscule, même si elle s'étend sur deux lignes.
}

12. Op. cit., p. 163. 